\title{
Crowdsourcing a crisis response for COVID-19 in oncology
}

\author{
Crowdsourcing efforts are currently underway to collect and analyze data from patients with cancer who are \\ affected by the COVID-19 pandemic. These community-led initiatives will fill key knowledge gaps to tackle \\ crucial clinical questions on the complexities of infection with the causative coronavirus SARS-Cov- 2 in the large, \\ heterogeneous group of vulnerable patients with cancer.
}

\section{Aakash Desai, Jeremy Warner, Nicole Kuderer, Mike Thompson, Corrie Painter, Gary Lyman and Gilberto Lopes}

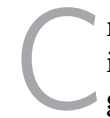
rowdsourcing is a model in which individuals or organizations obtain goods, services and ideas from a large, open community of internet users. The term, coined in 2006, is a combination of the words 'crowd' and 'outsourcing', and the process has become a commonplace phenomenon in the age of the internet ${ }^{1}$. In medicine, crowdsourcing began in earnest around 2010 in the fields of biomedical informatics and health-information technology ${ }^{2}$, with early implications for diagnostics and surveillance. Since then, crowdsourcing efforts have been burgeoning across public health, pharmacology, genetics, education ${ }^{3}$ and in various medical specialties such as oncology ${ }^{4}$. Such applications are seen at various levels of the healthcare infrastructure, spanning health promotion, research and maintenance. Healthcare tasks such as patient surveillance or monitoring, data processing, analysis and problem-solving have particularly reaped the benefits of crowdsourcing applications ${ }^{5}$.

\section{Crowdsourcing in oncology}

Crowdsourcing has been used increasingly in oncology since 2012, covering cancer types as varied as breast, skin, ovarian, cervical, colorectal, and prostate ${ }^{2}$. Currently, approximately $7 \%$ of all crowdsourcing initiatives in health map to the field of oncology ${ }^{5}$. The large pool of participants and time-efficient data collection that crowdsourcing affords, combined with lower costs and the potential for rapid innovation, make such initiatives valuable in solving many of the challenging questions in oncology. Indeed, one out of every three crowdsourcing studies in oncology is geared toward problem-solving, such as augmenting the work of trained professionals, developing protocols, randomized controlled trials and prognostic models, and assessing healthcare behaviors on a large scale ${ }^{2}$. Apart from academic collaborations, many biotechnology companies have invested in networks of crowdsourced data-driven research. For example, Sage Bionetworks, a Seattle-based biotechnology company, developed a registry for mapping cancer genomic data to clinical outcomes. The Myeloma Crowd Research Initiative, which uses a patient-driven approach to prioritize clinical-trial design and funding, exemplifies the formation of successful collaborations among patient advocates, physicians and patients through crowdsourcing and crowdfunding (a fundraising practice based on crowdsourcing $)^{6}$. More recently, a combination of artificial intelligence (AI) and crowdsourcing was described in radiation oncology as facilitating the development and clinical use of AI approaches, replicating some of the critical skills of highly trained physicians in radiation therapy at an ambitious scale. The use of AI could help improve cancer care on a global level by ensuring that the skills of expert clinicians are accessible by patients in countries with under-resourced healthcare systems?

Innovation is also occurring in terms of the development of workflows and tools for obtaining, sharing and analyzing oncology data. The development of a crowdsourcing framework for the annotation of medical datasets to simplify research and support workers in quickly analyzing information from complex datasets is such an example ${ }^{8}$. In addition, the DREAM Challenges crowdsourcing project has used secure technology to share portions of cancer clinical-trial data with crowdsourced teams to create prediction models while maintaining large validation sets behind an honest-broker platform ${ }^{9}$. This approach of 'bringing the data to the model' has increasingly allowed crowdsourced activity with highly sensitive data such as medical records and genomic datasets. When conducted in a manner that satisfies ethical considerations and privacy concerns, such an approach can be very beneficial to furthering research and patient care.

\section{Crowdsourcing during crises}

The utility of crowdsourcing through the power of social media has been evident in crisis monitoring, emergency planning and crisis management, social cohesion and research. The broad reach of social-media platforms gives access to the firsthand experiences of 'the many' and permits crowdsourcing and collaboration for the extensive mobilization of skills and the creation of networks, leadership and support systems, which allows problem-solving in record time. Crisis mapping was one of the first uses of social media in natural disasters. Ushahidi (https://www.ushahidi.com) is an open-source crisis-mapping platform originally developed in 2007 and used in Kenya, Mexico, Afghanistan and Haiti. It used Web 2.0 technologies to integrate data from sources such as phones, e-mail, web applications and social-media sites to map crisis areas as close to real time as possible and to make this available to the public, including to crisis-relief organizations ${ }^{10}$.

Crowdsourcing has been shown to be beneficial to disaster relief in many countries $^{11}$. For example, during the 2012 and 2013 Colorado wildfires, crowdsourcing played an important role in focusing resource allocation and disaster-relief efforts to severely affected areas. Crowdsourcing has also been used to instantaneously report health issues, such as cholera during the 2010 earthquake in Haiti, and dengue in Thailand and Indonesia ${ }^{12}$. Equally importantly, crowdsourcing during medical emergencies may also aid in elucidating disease presentation and modes and patterns of transmission; in identifying patients at higher risk and potential novel treatment options; and in increasing the speed with which a medical emergency is fought. For 
example, the e-mail update subscription service of the Program for Monitoring Emerging Diseases (ProMED-Mail) was credited with the detection of and resulting global response to the 2003 outbreak of the respiratory syndrome SARS months before the Chinese government's official report ${ }^{13}$.

\section{Crowdsourcing in the era of COVID-19}

The world is now in the midst of a pandemic of the respiratory syndrome COVID-19 ('coronavirus disease 2019'), caused by the coronavirus SARS-CoV-2 $2^{14}$. With over 2,400,000 diagnosed cases across 210 countries and territories around the world at the time of this writing, this is one of the largest medical disasters since the influenza pandemic of 1918.

As countries grapple with the lack of reliable and widely available COVID-19 testing capabilities, the absence of effective treatments and a looming shortage of personal protective equipment, they have been ramping up measures to prevent disease transmission, to protect healthcare workers and to increase healthcare-system capacity. This global health emergency has also spurred an unprecedented response by the biomedical research community aimed at identifying the mechanism of transmission, infection and immune response, case presentation, epidemic forecasting and treatments for this novel disease. However, despite the burgeoning number of publications (over 2,000 in the first 3 months after the first COVID-19 cases were reported in the Hubei province of China), most studies have been small and limited in their clinical scope and often raise more questions than the answers they provide. This is a consequence of the urgency with which the biomedical community is trying to respond to and disseminate knowledge about the pandemic. However, it has created an urgent need for larger, community-led projects to enable the identification of the main clinical challenges that need to be addressed and to provide rapid access to larger amounts of data that may point to the most promising applicable solutions in real time.

Various novel applications have already been developed as an emergency response to COVID-19, ranging from AI software that detects signs of pneumonia from lung CT scans to applications that aim to measure, model and decrease the spread of the disease by collecting information on symptoms and using smartphone wireless signals to crowd-map positive cases ${ }^{15}$. One such project, called 'CoEpi', attempts to bridge the gap between governmental public-health actions and individual attention to hygiene by using modern smartphone technology and cloud computing to empower people to anonymously track their own contacts and inform themselves about increased infection risks (https://www.coepi.org/). More recently, Harvard Medical School and Boston Children's Hospital collaborated on such a project in the USA, called 'COVID Near You'.

Beyond the use of crowdsourcing in emergency response and preparedness, healthcare professionals are also setting up crowdsourcing initiatives to understand the disease spectrum, risk factors and presentations and to optimize treatment strategies. Physicians across various specialties, ranging from hepatology to rheumatology and including oncology, have jumpstarted such crowdsourcing projects to serve their patient communities (Table 1).

\section{Crowdsourcing in COVID-19 and cancer} There remains a great deal of uncertainty about COVID-19 and its effects on people, especially the comorbid and the immunocompromised. Patients with cancer form a unique subset of people who are often both elderly and immunocompromised, may have substantial comorbidities and may be receiving treatment that frequently worsens immunosuppression and the risk of concomitant infections. Given the prevalence of cancer worldwide and the high transmissibility of COVID-19, there is a pressing need to understand the effects of this new infection and its associated and potentially serious outcomes specifically for patients with cancer.

Early data from China described a group of 18 patients with COVID-19 who had a history of cancer among a cohort of 1,590 patients with COVID-19 (1\% prevalence; $95 \%$ confidence interval (CI), $0.61-1.65 \%$ ), which was higher than the incidence of cancer in the overall Chinese population $(0.29 \%)^{16}$. A recent meta-analysis of 11 studies found that the overall pooled prevalence of cancer in patients with COVID-19 was 2.0\% (95\% CI, 2.0-3.0\%; $\left.\mathrm{I}^{2}=83.2 \%\right)^{17}$. Furthermore, patients with cancer had a higher risk of severe events (an endpoint defined as the percentage of patients being admitted to the intensive care unit who required invasive ventilation, or died) than that of patients without cancer (7 of 18 patients (39\%) versus 124 of 1,572 patients (8\%). respectively; $P=0.0003$ (Fisher's exact test)). A preprint study from China reported an infection rate of SARS-CoV-2 in patients with cancer as $0.79 \%$ ( 12 of 1,524 patients; $95 \% \mathrm{CI}$, $0.3-1.2 \%)^{18}$. Patients with cancer had a higher risk of SARS-CoV-2 infection (odds ratio, 2.31; 95\% CI, 1.89-3.02) than that of the community. Of the 1,524 patients with cancer, 228 (14.96\%) had non-small-cell lung cancer. A separate study from Italy reported a group of 355 comorbid patients with COVID-19 who died, of whom $72(20.3 \%)$ had active cancer ${ }^{19}$. These preliminary data provide some insights into the correlation between COVID-19 and cancer and indicate that more-intensive attention must be provided to patients with cancer, especially those with bone marrow or stem transplants, those with hematological malignancies and those in active treatment, given that they may be at higher risk.

However, given the small number of cases studied, the conclusions that can be reached remain tenuous. Moreover, there is currently no evidence and no clear recommendations on whether stopping or delaying chemotherapy, immunotherapy, radiation and adjuvant therapy and the use of supportive care such as hematopoietic growth factors would be advisable for patients with cancer. Some of the urgent questions that remain unanswered are as follows:

1. Are patients with cancer who contract COVID-19 at higher risk than that of otherwise healthy people?

2. Are patients with specific cancers or undergoing specific cancer therapies at higher risk than that of others?

3. Should adjuvant chemotherapy or elective surgery for stable patients with cancer be intentionally postponed as a prophylactic measure?

4. How do specific therapeutic modalities, such as immunotherapies, act in the context of COVID-19?

5. Should patients with cancer and cancer survivors be advised to use enhanced personal protection?

6. Would patients with cancer specifically benefit more from online medical counseling and online means for the identification and treatment of critical cases?

7. How should the effects of enforced quarantine on clinical trials be addressed, if it prevents attendance at hospital appointments and affects continuity of care?

More data need to be obtained and analyzed at a larger scale to provide definitive answers to these questions. Wider community collaboration clearly has an important role to play in addressing these pressing issues. Indeed, several crowdsourcing efforts are currently underway within oncology (Table 1). Among them, the American Society of Hematology Research Collaborative Registry for Hematological Malignancy aims to 
Table 1 | Crowdsourcing projects for COVID-19 in oncology and medicine

\begin{tabular}{|c|c|c|c|c|}
\hline Project & Specialty & Goal & Country & Registry or website url \\
\hline $\mathrm{CCC} 19$ & Oncology & $\begin{array}{l}\text { To collect information about patients with cancer } \\
\text { who have COVID-19, with the intent of using } \\
\text { and disseminating information to clinicians and } \\
\text { improve outcomes }\end{array}$ & International & https://ccc19.org/ \\
\hline $\begin{array}{l}\text { UK Coronavirus } \\
\text { cancer-monitoring } \\
\text { project }\end{array}$ & Oncology & $\begin{array}{l}\text { To collect information about prevalence of } \\
\text { COVID-19 with associated anonymized data } \\
\text { about site of disease and mortality }\end{array}$ & UK & $\begin{array}{l}\text { https://ukcoronaviruscancermonitoring. } \\
\text { com/ }\end{array}$ \\
\hline TERAVOLT & Oncology & $\begin{array}{l}\text { A global consortium designed to gather } \\
\text { information on patients with thoracic cancer } \\
\text { who have COVID-19, regardless of therapies } \\
\text { administered }\end{array}$ & International & N/A; participants join by e-mail \\
\hline $\begin{array}{l}\text { ASH RC COVID-19 } \\
\text { Registry for } \\
\text { Hematologic } \\
\text { Malignancy }\end{array}$ & $\begin{array}{l}\text { Hematology- } \\
\text { oncology }\end{array}$ & $\begin{array}{l}\text { To capture data on people who test positive } \\
\text { for COVID-19 and have been or are currently } \\
\text { being treated for hematological malignancies } \\
\text { and report near real-time observational data; } \\
\text { summaries will be reported for clinicians }\end{array}$ & International & $\begin{array}{l}\text { https://www.ashresearchcollaborative. } \\
\text { org/covid-19-registry }\end{array}$ \\
\hline ASCO Registry & Oncology & $\begin{array}{l}\text { To capture COVID-19 treatments and outcomes } \\
\text { as well as cancer treatment and outcomes, } \\
\text { including long-term effects for those who } \\
\text { recover from COVID-19 }\end{array}$ & USA & $\begin{array}{l}\text { https://www.asco.org/ } \\
\text { asco-coronavirus-information/ } \\
\text { coronavirus-registry }\end{array}$ \\
\hline $\begin{array}{l}\text { SECURE-SCD } \\
\text { Registry }\end{array}$ & Hematology & $\begin{array}{l}\text { To report on outcomes of COVID-19 in the } \\
\text { population of patients with sickle-cell disease }\end{array}$ & International & https://covidsicklecell.org/ \\
\hline $\begin{array}{l}\text { COVID-19 Global } \\
\text { Rheumatology } \\
\text { Alliance }\end{array}$ & Rheumatology & $\begin{array}{l}\text { To guide rheumatology clinicians in assessing } \\
\text { and treating patients with rheumatological } \\
\text { disease and in evaluating the risk of infection in } \\
\text { patients on immunosuppression }\end{array}$ & International & https://rheum-covid.org/ \\
\hline $\begin{array}{l}\text { SECURE-Cirrhosis } \\
\text { Registry }\end{array}$ & Hepatology & $\begin{array}{l}\text { To collect data on COVID-19 in patients with } \\
\text { chronic liver disease (with or without cirrhosis) } \\
\text { and after liver transplantation, regardless of the } \\
\text { severity of COVID-19 (including asymptomatic } \\
\text { patients detected through public health } \\
\text { screening) }\end{array}$ & $\begin{array}{l}\text { North or South } \\
\text { America, or China, } \\
\text { Japan and Korea }\end{array}$ & https://covidcirrhosis.web.unc.edu/ \\
\hline COVID-HEP Registry & Hepatology & $\begin{array}{l}\text { To collect data on patients with liver } \\
\text { disease or liver transplants who develop } \\
\text { laboratory-confirmed COVID-19 }\end{array}$ & European Union & https://www.covid-hep.net/ \\
\hline SECURE-IBD Registry & Gastroenterology & $\begin{array}{l}\text { To capture the natural history and outcomes of } \\
\text { children and adults with IBD in the context of } \\
\text { COVID-19 }\end{array}$ & International & https://covidibd.org/ \\
\hline COVID-19 in PID & Immunology & N/A & N/A & $\begin{array}{l}\text { https://www.surveymonkey.com/ } \\
\text { r/67RBPNZ? }\end{array}$ \\
\hline
\end{tabular}

ASH RC, American Society of Hematology Research Collaborative; ASCO, ASCO Survey on COVID-19 in Oncology; IBD, inflammatory bowel disease; PID, pelvic inflammatory disease; N/A, not available.

capture data on COVID-19-positive patients who have been or are currently being treated for hematological malignancies and to rapidly report conclusions on clinical practice and patient outcomes to clinicians, so that they may deliver appropriate care to their patients. A similar international effort focusing on thoracic cancers specifically is TERAVOLT ('thoracic cancers international COVID-19 collaboration'). The UK Coronavirus cancer-monitoring project is a clinician-led initiative that aims to study the prevalence of COVID-19 among patients with cancer and association of disease site on outcomes and mortality through comprehensive reporting, so that cancer care can continue to be provided where needed across the UK. In the USA, the recently launched American Association of Clinical Oncology (ASCO) Survey on COVID-19 in Oncology Registry aims to assess the impact of COVID-19 on patients with cancer and the provision of cancer care by collecting baseline and follow-up data on COVID-19 and cancer treatments and patient outcomes.

An additional, global crowdsourcing effort in oncology is the COVID-19 and Cancer Consortium (CCC19) and its cohort study tool, the web-based CCC19 Registry (Table 1). This consortium, which had its origins on Twitter, now comprises more than 90 cancer centers and other organizations all over the world. Healthcare providers (including pharmacy and nursing societies and patient advocates) from the USA, the European Union, Argentina, Canada and the UK have joined forces under the auspices of CCC19 to understand the unique effects of COVID-19 on patients with cancer. The CCC19 Registry involves a survey tool through which healthcare professionals can provide information on patients with cancer who have COVID-19. The type of information collected includes patient demographics, 
clinicopathological factors, cancer and COVID-19 diagnoses and treatments, and course of illness and outcomes, and also data about the healthcare professional. By rapidly collecting clinical and biological data about patients with cancer directly from the community, this initiative aims to improve the understanding of the scope, severity and related complications from COVID-19 in this uniquely heterogeneous and vulnerable patient population.

As the oncology community navigates the COVID-19 global health emergency and searches for answers to the many urgent clinical questions it poses, it is imperative that data-driven decisions be made by those caring for patients. Crowdsourcing to quickly accrue, analyze and disseminate data from large numbers of patients by tapping into freely available resources provides a unique opportunity for tackling the complexities of COVID-19 in patients with cancer. The community efforts discussed here bring together an unprecedented number of collaborators and leverage their expertise and resources to fill key knowledge gaps within the field of oncology in this ongoing medical crisis, aiming to rapidly address the problems with the most urgent need for solutions.

\section{Aakash Desai', Jeremy Warner ${ }^{2,3}$, \\ Nicole Kuderer ${ }^{4}$, Mike Thompson ${ }^{5}$, \\ Corrie Painter ${ }^{6}$, Gary Lyman ${ }^{7,8}$ and \\ Gilberto Lopes ${ }^{9 凶}$}

${ }^{1}$ Department of Medicine, University of Connecticut, Farmington, CT, USA. ${ }^{2}$ Department of Medicine, Division of Hematology and Oncology, Vanderbilt University, Nashville, Tennessee, USA. ${ }^{3}$ Department of Biomedical Informatics, Vanderbilt University, Nashville, TN, USA. ${ }^{4}$ Advanced Cancer Research Group, Seattle, WA, USA. ${ }^{5}$ Aurora Research Institute, Aurora Health Care, Milwaukee, WI, USA. ${ }^{6}$ Broad Institute of Harvard and MIT, Cambridge, MA, USA. ${ }^{7}$ Department of Medicine, University of Washington, Seattle, WA, USA. ${ }^{8}$ Hutchinson Institute for Cancer Outcomes Research, Fred Hutchinson Cancer Research Center, Seattle, WA, USA. ${ }^{9}$ Division of Hematology and Medical Oncology, Department of Medicine, Miller School of Medicine, University of Miami, Sylvester Comprehensive Cancer Center, Miami, FL, USA.

凶e-mail: glopes@med.miami.edu

Published online: 21 April 2020

https://doi.org/10.1038/s43018-020-0065-z
References

1. Howe, J. Wired 14, 1-4 (2006)

. Lee, Y. J., Arida, J. A. \& Donovan, H. S. Cancer Med. 6 2595-2605 (2017).

3. Blackwell, K. A., Travis, M. J., Arbuckle, M. R. \& Ross, D. A. Med. Educ. 50, 576 (2016)

4. Yang, S., Wang, S. J. \& Ji, Y. Contemp. Clin. Trials 45(Pt B), 426-434 (2015)

5. Créquit, P., Mansouri, G., Benchoufi, M., Vivot, A. \& Ravaud, P. J. Med. Internet Res. 20, e187 (2018)

6. Butcher, L. Oncology Times 37, 18-20 (2015)

7. Mak, R. H. et al. JAMA Oncol. 5, 654-661 (2019).

8. Ye, C. et al. AMIA Jt. Summits Transl. Sci. Proc. 2017, 273-280 (2018)

9. Guinney, J. et al. Lancet Oncol. 18, 132-142 (2017).

10. Gao, H., Barbier, G. \& Goolsby, R. IEEE Intell. Syst. 26, 10-14 (2011).

11. Riccardi, M. T. Int. J. Disaster Risk Reduct. 20, 123-128 (2016).

12. Boulos, M. N. K. et al. Int. J. Health Geogr. 10, 67 (2011).

13. Yong, E. Br. Med. J. 343, d4117, https://doi.org/10.1136/ bmj.d4117 (2011).

14. Centers for Disease Control and Prevention. https://www.cdc. gov/coronavirus/2019-ncov/cases-updates/summary.html (accessed 14 March 2020)

15. Knight, W. W. Wired https://www.wired.com/story/ phones-track-spread-covid19-good-idea/ (2020).

16. Liang, W. et al. Lancet Oncol. 21, 335-337 (2020).

17. Desai, A., Sachdeva, S., Parekh, T. \& Desai, R. JCO Global Oncology 6, 557-559 (2020).

18. Yu, J., Ouyang, W., Chua, M. L. \& Xie, C. medRxiv https://doi.org/10.1101/2020.02.22.20025320 (2020)

19. Onder, G., Rezza, G. \& Brusaferro, S. J. Am. Med. Assoc. https://doi.org/10.1001/jama.2020.4683 (2020).

Competing interests

The authors declare no competing interests. 